\title{
EL CRECIMIENTO URBANO DE BENIDORM SEGÚN LOS EXPEDIENTES DE OBRAS (1950-1970)
}

\author{
Gabino-A. Martín-Serrano Rodríguez \\ Departamento de Geografía Humana \\ Universidad de Alicante
}

\section{RESUMEN}

La ciudad de Benidorm ha experimentado grandes cambios en el último medio siglo. En 1950 aún conservaba un sistema productivo centrado en las actividades agrícolas, pero a partir de ese año, tendrán lugar procesos de diversa índole que transformarán la estructura social y productiva del municipio. Entre estos procesos cabe destacar el crecimiento urbano, que significó no sólo una expansión superficial de la trama urbana, sino un cambio radical en la manera de hacer ciudad. El periodo de tiempo entre 1950 y 1970 es fundamental a la hora de entender estos cambios, ya que se puede considerar una etapa de transición en la que se propusieron diferentes modelos de ciudad y se establecieron las claves para la formación de la ciudad actual.

Palabras clave: Benidorm, crecimiento urbano por licencias de obras.

\section{RÉSUMÉ}

La ville de Benidorm a connu de grands changements durant ce dernier demi siècle. En 1950 la ville conservait encore un système productif basé sur les activités agricoles, mais à partir de cette année là, des processus divers ont transformé la structure sociale et productive de la commune. Parmis ces processus il faut mettre l'accent sur la croissance urbaine, qui a représenté non seulement une expansion superficielle du tracé urbain, mais aussi un changement radical dans la conception de la ville. La période comprise entre 1950 et 1970 est fondamentale pour pouvoir comprendre ces changements, étant donné que l'on peut la considérer comme une étape de transition dans laquelle on a proposé divers modèles d'urbanisation et on a établi les règles pour la formation de la ville actuelle.

Mots clés: Benidorm, croissance urbaine et permis de construction.

\section{El cambio socioeconómico de Benidorm durante el periodo 1950-1970}

En aproximadamente cincuenta años Benidorm ha pasado de ser un pequeño núcleo costero Mediterráneo con 2.787 habitantes de derecho en 1950 (INE, Censo, 1950), con un 


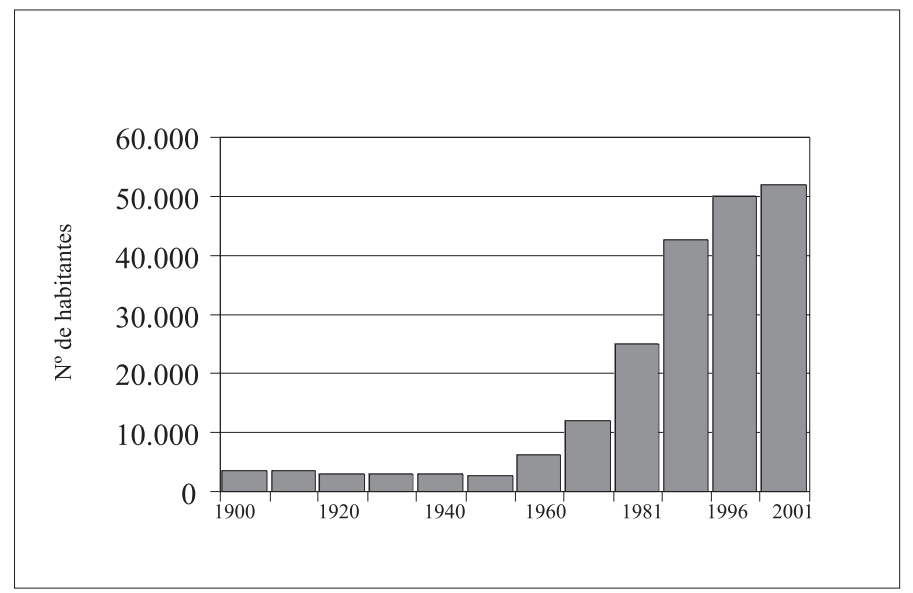

FiguRA 1. Benidorm. Evolución de la población de derecho (1900-2001)

Fuente: INE, Censos de población (1900, 1910, 1920, 1930, 1940, 1950, 1960, 1970, 1981, 1991 у 2001) у Padrón Municipal de habitantes (1996).

sector productivo caracterizado entonces por su dedicación agraria (aproximadamente el $60 \%$ de la población vivía del sector productivo primario), a ser una Ociurbe (CosTA MAs, 1997) con 51.873 habitantes en 2001 (INE, Censo, 2001), y con un 79’5 \% de su población activa empleada en el sector productivo terciario.

La evolución demográfica del municipio (fig. 1) muestra una clara ruptura en 1960: hasta entonces Benidorm se caracteriza por un modelo demográfico claramente emigratorio (aunque mantiene su población a lo largo de la primera década del siglo); el desarrollo turístico marcará claramente el paso desde el anterior modelo demográfico por otro de carácter inmigratorio. El éxodo rural producido en esos años desde los lugares más deprimidos económicamente a los que mostraban mayor dinamismo, será factor fundamental en la evolución de Benidorm.

En los años '50, antes de que Benidorm se orientara definitivamente hacia el turismo, la principal ocupación a que se dedicaban sus habitantes era la agricultura, en su mayoría de secano. La mayor parte del suelo puesto en uso en el término municipal estaba dedicado a tierras de labor, predominando los cereales, viñas y olivar, y en menor medida hortalizas en pequeñas huertas. Otra ocupación importante de los benidormíes era embarcarse como marino en la Marina Mercante. En palabras de M. Gaviria, J. M. Iribas, F. Sabbah y J. R. Sanz Arranz (1977): «... Benidorm era un pueblo marinero cuyos hombres navegaban en las compañías navieras españolas y extranjeras y emigraban a la Almadraba. La agricultura, de secano principalmente [...], era un pueblo de economía acostumbrada a la vida dura. Tenía tierras, pero no tenía apenas regadíos [...] Benidorm era un pueblo de marinos, no de pescadores». Benidorm no disponía de puerto pesquero, ni por tanto de una flota pesquera capacitada que empleara a un porcentaje importante de su población. La principal actividad de pesca local se realizaba con carácter de autosuministro, con una pequeña flota pesquera compuesta por 10 ó 14 barcos (Ayuntamiento de Benidorm, 1955). En este sentido, Benidorm no se diferenciaba económica ni socialmente, en términos generales, de los muchos municipios españoles que en los '50 empezaban a sufrir de forma acusada el fenómeno del éxodo rural. Una agricultura que perdía constantemente valor frente a los productos de la ciudad, con claros problemas estructurales, es el hilo conductor de este 
fenómeno. El proceso es mucho más virulento en otras partes de la península, sobre todo donde el número de jornaleros es muy grande frente al de propietarios. En Benidorm, tanto por la menor intensidad de este último factor en los motivos del éxodo rural, como por el encadenamiento de decisiones precisas y lo coyuntural de la moda turística, el descenso de la población invierte su tendencia.

Ya antes de 1950 se habían venido practicando actividades turísticas pero no de forma sistemática y planificada, sin influir de manera decisiva en la economía local. Será aproximadamente hacia la mitad de la década de los '50, como señalan varios autores (VERA, J. F., 1987; Gaviria, M., 1977; y Ayuntamiento De Benidorm, 1955), a partir de la aprobación del Plan General de Ordenación Urbana de Benidorm el 18 de abril de 1956, cuando se empieza a hacer esbozos del modelo social, económico y espacial que se desea para Benidorm. La idea actual de un Benidorm dedicado plenamente al turismo de masas no surgió de forma espontánea y nítida. El proyecto de futuro en esos momentos se asemejaba mucho más a lo que sería hoy en día ciertos lugares de la vecina Calpe; una ciudad jardín de casas de planta baja o dos plantas, tipo chalet, bien construidas individualmente, bien planificadas y agrupadas en urbanizaciones. Por tanto se pensó en un modelo general de ciudad jardín a implantar en el suelo urbanizable fuera del casco urbano; sin embargo, tras sucesivas modificaciones del Plan General, aumentarán los volúmenes permitidos de edificación y no se limitará la altura máxima permitida. A partir de 1963 comienza a construirse un nuevo modelo de ciudad, de bloques abiertos, falso open planning por las bandejas y los locales comerciales construidos en sus bajos (Costa MAs, 1997) y que serán fundamentales para comprender el proceso constructivo en el ensanche de levante y en otros puntos localizados de la ciudad (VERA ReBollo, 1987).

Los cambios en la política urbana de Benidorm tienen efectos muy importantes, aparte de los ocurridos en la morfología urbana. Estos cambios llevan implícito no sólo un nuevo modelo de hacer ciudad, sino la consideración de diferentes modos de organización social. Pero estos dos factores no han de ser tenidos en cuenta por separado, pues los diferentes elementos que los componen forman parte de un complejo sistema de interacción. Podemos afirmar que el espacio social en un momento dado es resultado de una determinada formación social - de la lucha entre los distintos sistemas productivos que la componen, del conflicto de objetivos entre las distintas facciones, de las estrategias y medios empleados para llevarlos a efecto-, no es menos cierto que el espacio social dado puede constreñir y definir el modo de vida de muchas personas en un lugar y momento determinado, así como delimitar las posibilidades de acción, sobre todo en función de su posición tanto en el espacio como en la jerarquía social (SÁnchez, 1981; 1991).

Actualmente la ciudad de Benidorm está caracterizada por una clara monoespecialización turística, que se extiende a otras varias ramas como las actividades inmobiliarias, comerciales, administrativas, etc. ligadas a esta forma principal de producción. Dentro de este contexto el mercado laboral está formado por una mayoría de asalariados que de una u otra manera trabajan en el sector turístico. Los servicios y equipamientos dedicados a la atención de la población residente poseen muchas deficiencias, mientras que forman excedente los ideados con vistas a la atención del turista.

\section{Los ritmos de construcción}

\subsection{Número y naturaleza de las licencias de obras}

El ritmo de construcción en Benidorm desde 1950 a 1970 ha sido estudiado a través de las solicitudes de licencia de obra. Estas solicitudes varían mucho interanualmente, pero la 
tendencia general a lo largo del periodo es creciente en número de solicitudes. Así, si consideramos el número de expedientes totales (Cuadro 1), en 1951 son 72, en 1952 ascienden a 129, mientras que en 1953 y 1954 están muy por debajo de 100 (49 y 69 respectivamente), pero en 1955 el número es de 231.

La interpretación de la tendencia es más fácil diferenciando entre los expedientes de nueva planta y los expedientes de adición:

a) 1951-1955: este primer quinquenio está caracterizado por un número mayor de solicitudes de expedientes de adición. Se corresponden en su mayoría con reformas y arreglos en las casas de la población autóctona. El número de expedientes de adición está por debajo de 100 en los años 1951, 1953 y 1954 (66, 33 y 46 respectivamente) pero en 1952 son 121 las solicitudes y 193 en 1955. Los expedientes de nueva planta son solicitados para la construcción de viviendas particulares por los usuarios, así como para la construcción de medios de producción ligados a la agricultura. En cada año del quinquenio su número no supera las 50 solicitudes, y responden principalmente a las necesidades básicas de la población autóctona.

\section{Cuadro 1}

BENIDORM (1951-1969). NÚMERO DE EXPEDIENTES DE NUEVA PLANTA, DE ADICIÓN Y TOTAL POR AÑO

\begin{tabular}{|l|c|c|c|}
\hline Año & Expedientes de Nueva Planta & Expedientes de Adición & Total \\
\hline 1951 & 6 & 66 & 72 \\
\hline 1952 & 8 & 121 & 129 \\
\hline 1953 & 16 & 33 & 49 \\
\hline 1954 & 23 & 46 & 69 \\
\hline 1955 & 38 & 193 & 231 \\
\hline 1956 & 54 & 181 & 235 \\
\hline 1957 & 45 & 41 & 86 \\
\hline 1958 & 54 & 237 & 291 \\
\hline 1959 & 58 & 40 & 98 \\
\hline 1960 & 27 & 28 & 55 \\
\hline 1961 & 83 & 61 & 144 \\
\hline 1962 & 103 & 70 & 173 \\
\hline 1963 & 122 & 76 & 198 \\
\hline 1964 & 142 & 91 & 233 \\
\hline 1965 & 45 & 35 & 80 \\
\hline 1966 & 74 & 75 & 149 \\
\hline 1967 & 40 & 62 & 102 \\
\hline 1968 & 85 & 72 & 157 \\
\hline 1969 & 97 & 59 & $\mathbf{2 7 0 7}$ \\
\hline Total & $\mathbf{1 1 2 0}$ & & \\
\hline
\end{tabular}

Fuente: Archivo del Ayuntamiento de Benidorm, Licencias de Obras, 1951-1959. Elaboración propia. 
b) 1956-1960: En 1956 y 1958 se registran valores por encima de las 200 solicitudes (235 y 291 respectivamente), pero en 1957 y 1959 los valores bajan de las 100 (86 y 98) y en 1960 sólo son 55. La variación interanual vuelve a ser muy grande, debido sobre todo a la influencia de los expedientes de adición, que todavía es muy importante. En 1958 el número de solicitudes es superior a 200 (237) y en 1956 se acerca mucho a ese valor (181). En el resto de años del quinquenio el número de solicitudes no supera las 50 licencias.

b.1) Expedientes de Adición: a partir de 1960 los expedientes de adición pierden importancia respecto a los expedientes de nueva planta (cuadro 1). Así mismo, en 1960 se produce un cambio en la naturaleza de los expedientes de adición. Hasta ese año son destinados principalmente a reformas de los inmuebles (cuadro 2), pero a partir de 1960 son utilizados para la ampliación de los inmuebles y para el cambio de uso y distribución. Estas últimas funciones están más relacionadas con un territorio que incrementa el valor del terreno, lo que posibilita la reinversión en los espacios construidos y la apropiación de nuevas plusvalías. Esto es posible también gracias a los cambios acaecidos en la ley del

Cuadro 2

BENIDORM (1951-1969). EXPEDIENTE DE ADICIÓN POR AÑO SEGÚN REFORMA, AMPLIACIÓN Y CAMBIO DE DISTRIBUCIÓN

\begin{tabular}{|c|c|c|c|}
\hline Año & Reforma & Ampliación & Cambio de distribución y uso \\
\hline 1951 & 65 & 4 & 1 \\
\hline 1952 & 117 & 17 & 1 \\
\hline 1953 & 32 & 2 & - \\
\hline 1954 & 32 & 15 & - \\
\hline 1955 & 175 & 19 & 3 \\
\hline 1956 & 159 & 20 & 9 \\
\hline 1957 & 14 & 32 & - \\
\hline 1958 & 214 & 19 & 9 \\
\hline 1959 & 24 & 9 & 2 \\
\hline 1960 & 8 & 14 & - \\
\hline 1961 & 19 & 29 & 3 \\
\hline 1962 & 27 & 23 & 1 \\
\hline 1963 & 17 & 27 & 5 \\
\hline 1964 & 19 & 22 & 4 \\
\hline 1965 & 8 & 23 & 3 \\
\hline 1966 & 12 & 43 & 12 \\
\hline 1967 & 16 & 39 & - \\
\hline 1968 & 17 & 31 & 5 \\
\hline 1969 & 16 & 46 & 2 \\
\hline Total & 991 & 434 & 60 \\
\hline
\end{tabular}

Fuente: Archivo del Ayuntamiento de Benidorm, Licencias de Obras, 1951-1969. Elaboración propia. 
suelo y en los documentos de ordenación del espacio urbano (aumentos del volumen permitido a partir de 1960 en el PGOU). Hasta 1960, los años con número de solicitudes de reforma por encima de 100 eran cuatro (117 en 1952, 175 en 1955, 159 en 1956 y 214 en 1958). El resto de años los valores están por debajo (65 en 1951, 32 en 1953 y 1954, 14 en 1957, 24 en 1959 y 8 en 1960). El número de solicitudes de reforma de 1959 y 1960 ya muestran el cambio de naturaleza en los expedientes de adición, pues después de 1960 el número de solicitudes está siempre por debajo de 20. Una dinámica muy distinta siguen los expedientes de adición dedicados a la ampliación y cambio de distribución de los inmuebles, pues su número regulariza y gana peso a partir de 1960 (cuadro 2), sobre todo los de ampliación.

b.2) Expedientes de Nueva planta: los expedientes de nueva planta (en el quinquenio 1956-1960) aumentan su número, aunque con diferencias intercensales. Estos cambios se deben a la aparición de nuevas dinámicas económicas y sociales en Benidorm. Así, las solicitudes de licencias de obras se hacen tanto para cubrir las necesidades básicas de la población residente, como para crear mercancías. Estas serán adquiridas por personas con diferentes objetivos, incrementando la demanda y con ello los beneficios obtenidos por los promotores. Este cambio ocurre mucho antes, como hemos visto, en los expedientes de nueva planta que en la naturaleza de los expedientes de adición. El cambio en la naturaleza de los expedientes de adición se debe principalmente a los cambios en las características de ciertas variables urbanísticas ocurridas a partir de 1960 (modificaciones del PGOU) y al creciente interés de agentes con poca capacidad de actuación pero que ven en los expedientes de ampliación la posibilidad de incrementar sus rentas. Sin embargo, los expedientes de nueva planta presentan una tendencia a crecer desde 1951, debido no solamente a la construcción de viviendas principales por parte de los usuarios, sino también a la construcción de chalets y viviendas secundarias por parte de inmigrantes y turistas que buscan en Benidorm un lugar tranquilo y agradable. A partir de 1960 las pautas cambian. La construcción de viviendas por parte de los autóctonos de Benidorm para su uso directo es menor en proporción a las construidas para albergar a los foráneos (tanto trabajadores como los que buscan ocio). Cambia también por tanto la tipología urbanística de estas construcciones. Hasta 1960 las edificaciones destinadas a inmigrantes eran principalmente chalets destinados al ocio y al descanso. A partir de 1960 los chalets disminuirán en proporción, destinados a la clase media-alta y localizados siempre alejados de la ciudad. Los edificios residenciales colectivos destinados a acoger a los inmigrantes trabajadores crecerán en número, así como los edificios y bloques de apartamentos destinados a funciones turísticas.

c) 1961-1965: el número de expedientes totales en este quinquenio está siempre por encima de los 100 expedientes (144 en 1961, 173 en 1962 y 198 en 1963), y en 1964 se alcanzan 233. Sin embargo, en 1965 se produce un gran descenso, con tan solo 80 expedientes totales, adelantando la caída producida en el cuatrienio de 1966-1969. Los expedientes de adición muestran valores por debajo de 100 (61 en 1961, 70 en 1962, 76 en 1963, 91 en 1964 y 35 en 1965), representados en gran medida por expedientes de ampliación. Los expedientes de nueva planta siguen su dinámica de crecimiento, pues aunque en 1961 aún están por debajo de 100 (83), en 1962, 1963 y 1964 sobrepasan el centenar (103, 122 y 142 respectivamente). El año 1965 (con 45 expedientes de nueva planta) inicia la crisis de expedientes de nueva planta del siguiente cuatrienio (1966 a 1969).

d) 1966-1969: en este periodo el número total de los expedientes supera en todos los años 100 (149 en 1966, 102 en 1967, 157 en 1968 y 156 en 1969), pero es en los expedientes de nueva planta donde se observa un frenazo en la actividad constructiva. En 
ningún año el número de expedientes de nueva planta es superior a 100 (74 en 1966, 40 en 1967, 85 en 1968 y 97 en 1969), mientras que en el periodo anterior sobrepasaban esa cifra holgadamente. Los expedientes de adición siguen la misma dinámica de años pasados (75 en 1966, 62 en 1967, 72 en 1968 y 59 en 1969). En tiempos de crisis o cuando el margen de beneficio (incluidas las plusvalías y excedente apropiado) no cumple las expectativas de los agentes promotores, se produce una reducción en las solicitudes de nueva planta. Esto se debe a que es preferible reservar los solares no edificados para aumentar los beneficios que se puedan obtener de su construcción. De esta manera, los esfuerzos se dirigen a reinvertir en lo ya construido y a la edificación de nueva planta en terrenos de poco valor y alejados de la ciudad. Los agentes buscan de esta manera crear las condiciones adecuadas para la posterior construcción en los solares de mayor valor.

Estas dinámicas se observan mejor si consideramos el número total de expedientes por quinquenio. Así, el número de expedientes tanto de nueva planta como de adición para el quinquenio 1951-1955 es de 550, en el quinquenio de 1956 a 1960 es de 765, de 828 en 1961-1965 y de 564 en el cuatrienio 1966-1969. El número de expedientes de nueva planta en el periodo 1951-1955 es de 91, de 238 en 1956-1960, de 495 en 1961-1965 y de 296 en el cuatrienio 1966-1969. El número de expedientes de adición en el quinquenio 1951-1955 es de 495, de 527 en 1956-1960, de 333 de 1961 a 1965 y de 268 en el cuatrienio 19661969. Así, el número total de expedientes crece de manera importante en los tres primeros quinquenios, mientras que en el último cuatrienio descienden. Los expedientes de nueva planta crecen lentamente en los dos primeros quinquenios, alcanzan un número importante en 1961-1965 para disminuir en el último cuatrienio debido al aumento de viviendas y apartamentos por expediente y a no reunirse las condiciones óptimas para la construcción. Los expedientes de adición parten de valores altos en los dos primeros quinquenios debido a la reutilización del parque inmobiliario construido y descienden progresivamente en el resto de periodos debido a la mayor importancia de la obra nueva y los expedientes de ampliación. Por tanto se constata un interés creciente en la construcción de parque inmobiliario nuevo, pese al descenso en el último cuatrienio.

\subsection{La altura de los edificios}

a) Expedientes de nueva planta

a.1) 1951-1960: la altura del parque inmobiliario, expresada a través del número de plantas, cambia a lo largo del periodo, debido a las causas apuntadas anteriormente. En este sentido, desde 1951 a 1960 la construcción de edificios de una sola planta (cuadro 3) se corresponde con la construcción de viviendas principales por la población autóctona de Benidorm y de chalets y viviendas secundarias para inmigrantes y turistas. Las edificaciones de dos plantas son muy importantes en este primer periodo. Contienen normalmente una única vivienda y poseen las mismas funciones que las construcciones de una sola planta. Conforme la dinámica urbana y las normas del PGOU van permitiendo aumentos de la superficie construida y de volumen, estos edificios se dividirán en varias viviendas y serán ampliados. Así vemos como en el periodo mencionado los expedientes de adición destinados a aumentar una sola planta (debido a los recursos disponibles para construir) son mayoría.

Hasta 1960 los edificios de tres a seis plantas no son muy numerosos, pero ya están presentes. Se materializan en hoteles y pequeños edificios de apartamentos y residenciales. Conforme aumentan los volúmenes permitidos en numerosos sectores del municipio, estos edificios irán ampliándose por plantas hasta alcanzar el máximo permitido. En cuanto a los 
edificios de siete y más plantas, en este periodo todavía no se ha solicitado ningún expediente. Esto es debido en parte a que las normas del PGOU de 1956 apenas han sido modificadas, y todavía no se ha autorizado la construcción libre en altura con limitación de volumen.

a.2) 1960-1969: a partir de 1960 el número de licencias de una planta se mantiene constante, debido sobre todo a la importancia del turismo residencial, que poco a poco irá perdiendo terreno respecto al turismo de masas y la residencia laboral. El número de solicitudes de dos plantas incluso decrece, mientras que los edificios de tres a seis plantas aumenta de forma significativa en los primeros años (34 en 1961, 48 en 1962, 44 en 1963 y 49 en 1964), para disminuir al final del periodo. La no limitación de altura con restricciones del volumen redactadas en las Modificaciones del PGOU de 1956, tras pequeñas

Cuadro 3

BENIDORM (1951-1969). NÚMERO DE EXPEDIENTES DE NUEVA PLANTA Y ADICIÓN SEGÚN NÚMERO DE PLANTAS DEL EDIFICIO

\begin{tabular}{|c|c|c|c|c|c|c|c|c|}
\hline \multirow[b]{3}{*}{ Año } & \multicolumn{8}{|c|}{ Expedientes } \\
\hline & \multicolumn{5}{|c|}{ Nueva planta } & \multicolumn{3}{|c|}{ Adición } \\
\hline & $1 P$ & $2 \mathrm{P}$ & 3P-6P & 7P-11P & $+11 \mathrm{P}$ & $1 P$ & $2 P$ & 3P y más \\
\hline 1951 & 3 & 3 & - & - & - & - & - & 1 \\
\hline 1952 & 4 & - & 1 & - & - & 9 & 1 & - \\
\hline 1953 & 9 & 7 & - & - & - & - & 1 & - \\
\hline 1954 & 1 & 9 & 6 & - & - & 10 & 3 & - \\
\hline 1955 & 4 & 10 & 9 & - & - & 13 & 2 & - \\
\hline 1956 & 4 & 15 & 11 & - & - & 10 & 6 & - \\
\hline 1957 & 26 & 11 & 7 & - & - & 20 & 2 & - \\
\hline 1958 & 35 & 8 & 11 & - & - & 10 & 2 & 1 \\
\hline 1959 & 18 & 11 & 19 & - & - & 11 & 1 & - \\
\hline 1960 & 16 & 3 & 7 & - & - & 7 & 3 & - \\
\hline 1961 & 24 & 7 & 34 & 2 & - & 12 & 2 & - \\
\hline 1962 & 37 & 8 & 48 & 5 & 1 & 18 & 3 & 1 \\
\hline 1963 & 28 & 14 & 44 & 7 & 6 & 19 & 5 & 1 \\
\hline 1964 & 35 & 9 & 49 & 19 & 12 & 21 & 8 & 4 \\
\hline 1965 & 9 & 2 & 19 & 10 & 6 & 4 & 2 & 3 \\
\hline 1966 & 18 & 2 & 27 & 13 & 9 & 13 & 5 & 5 \\
\hline 1967 & 20 & 4 & 5 & 10 & 1 & 12 & 2 & 4 \\
\hline 1968 & 34 & 4 & 16 & 18 & 7 & 9 & 4 & 8 \\
\hline 1969 & 37 & 6 & 15 & 18 & 22 & 6 & 4 & 16 \\
\hline Total & 362 & 133 & 328 & 102 & 64 & 204 & 56 & 44 \\
\hline
\end{tabular}

Fuente: Archivo del Ayuntamiento de Benidorm, Licencias de Obras, 1951-1969. Elaboración propia. 
modificaciones anteriores, en 1961 posibilita la construcción de más de 7 alturas. Así, a partir de 1961 ya existen solicitudes de edificios entre 7 y 11 plantas, y de más de 11 plantas a partir de 1962. El número creciente de solicitudes de este tipo hace que las solicitudes de entre 3 y 6 plantas disminuyan, centrándose sobre todo en el ensanche del casco histórico. Los edificios más altos se localizarán en los sectores más turísticos y donde las plusvalías son máximas, como en el Ensanche de Levante y ciertos sectores de la playa de poniente.

\section{b) Expedientes de adición}

En cuanto a la ampliación de plantas a través de expedientes de adición, a partir de 1960 las ampliaciones de 1 y 2 plantas se mantienen constantes. Sin embargo, la ampliación de tres plantas, a partir de 1961, empieza a ser importante sobre todo a través de la creación de anexos a los edificios ya existentes. En esencia es la creación de nueva construcción disimulada a través de expedientes de adición. Este fenómeno será muy importante en los hoteles, aunque también se dan casos en bloques de apartamentos. En cuanto a los edificios residenciales, suelen verse más afectados por la ampliación de plantas.

\section{La superficie territorial utilizada y la superficie construida}

A partir de 1950 Benidorm comienza a experimentar grandes cambios que afectarán a la manera de hacer ciudad. Así, antes de 1950 la construcción de ciudad se debía principalmente a promotores-usuarios. La superficie territorial consumida era pequeña y cercana a la ciudad compacta, salvo en los casos en que se construían casas de campo y equipamientos para las labores agrícolas. Así mismo, la superficie construida no daba lugar a grandes densidades edificatorias, y pese a que existía un continuo urbano, no era denso. Desde 1950 la superficie territorial utilizada para la construcción irá en ascenso, al igual que la superficie construida y la densidad edificatoria. Esta será más alta en los barrios de trabajadores, mientras que en los centros turísticos, pese a la visión de grandes torres, las densidades permanecerán más bajas y la ventilación será mucho mayor. El incremento del valor del suelo obligará en los casos posibles (como en viviendas de inmigrantes con pocos recursos) a aprovechar al máximo el volumen permitido.

En 1951 las solicitudes de expedientes de nueva planta arrojaban un consumo de 920 $\mathrm{m}^{2}$ de solar (Cuadro 4). Estas cifras, no dejarán de incrementarse, salvo en los años 1957, 1960 y en el quinquenio 1965-1969. En 1959 son 115.612 los metros cuadrados solicitados, y en 1964 se llega al máximo del periodo con $480.688 \mathrm{~m}^{2}$. La superficie de solares solicitada acumulada llega a ser de $2.663 .133 \mathrm{~m}^{2}$ en 1969 . Lo mismo ocurre con la superficie construida de los proyectos solicitados. Las solicitudes de 1951 tan solo contemplan $184 \mathrm{~m}^{2}$, mientras que en 1964 es de $188.612 \mathrm{~m}^{2}$. En cuanto a la superficie construida acumulada, evoluciona de $184 \mathrm{~m}^{2}$ en 1951 a $661.025 \mathrm{~m}^{2}$ en 1969. Estas cifras están muy por debajo de las reales. Esto es debido a que la mayor parte de los expedientes no muestran el dato o lo presentan de forma errónea, y no se ha hecho extrapolación. Este ocultamiento de datos es más evidente cuando comparamos estos datos con el cruce de las variables superficie del solar-superficie construida (Cuadro 7). Los mismos problemas aparecen con la superficie del solar, pero es mucho menor el número de casos y las cifras se aproximan más a la realidad. 
a) Evolución del tamaño del solar

La evolución del tamaño del solar en el periodo de 1951 a 1969 (Cuadro 5) nos muestra el incremento del interés constructivo. Desde 1951 a 1960 predomina el uso de parcelas pequeñas, hasta los $800 \mathrm{~m}^{2}$. Así, durante el periodo el número de solicitudes de obras en solares de menos de $100 \mathrm{~m}^{2}$ ha sido de 28, de 63 en los solares de 101 a $200 \mathrm{~m}^{2}$, de 19 en los solares de 201 a $400 \mathrm{~m}^{2}$, y de 61 en los solares de 401 a $800 \mathrm{~m}^{2}$. El mayor número de solicitudes se centra en los solares de 101 a $200 \mathrm{~m}^{2}$ y de 401 a $800 \mathrm{~m}^{2}$. En total estas solicitudes suman 171 expedientes. Los solares entre los 801 y los $1.200 \mathrm{~m}^{2}$ suman 62 solicitudes, 30 en los solares de 1.201 a $2.000 \mathrm{~m}^{2}$ y de más de $2.000 \mathrm{~m}^{2}$. En total suman 122 solicitudes. El elevado número de solicitudes en este tipo de solares se debe al incremento de su uso a partir del año 1957, que seguirá creciendo en el siguiente periodo, sobre todo en las parcelas más grandes.

Las edificaciones en solares pequeños se corresponden con viviendas particulares en el casco histórico o cercanas a él. Se empiezan a construir los primeros edificios residenciales en el ensanche del casco, aunque de tamaño y número de viviendas reducidos. La construc-

$$
\text { Cuadro } 4
$$

BENIDORM (1951-1969). SUPERFICIE DEL SOLAR CONSUMIDA Y SUPERFICIE CONSTRUIDA POR AÑO

\begin{tabular}{|c|r|r|r|c|}
\hline $\mathbf{A n ̃ o}$ & $\begin{array}{c}\text { Superficie solar } \\
\left(\mathbf{m}^{2}\right)\end{array}$ & $\begin{array}{c}\text { Superficie solar } \\
\text { acumulada }\left(\mathbf{m}^{2}\right)\end{array}$ & $\begin{array}{c}\text { Superficie } \\
\text { construida }\left(\mathbf{m}^{2}\right)\end{array}$ & $\begin{array}{c}\text { Superficie construida } \\
\text { acumulada }\left(\mathbf{m}^{2}\right)\end{array}$ \\
\hline $\mathbf{1 9 5 1}$ & 920,00 & 920,00 & 183,79 & 183,79 \\
\hline $\mathbf{1 9 5 2}$ & 950,18 & $1.870,18$ & $3.258,00$ & $3.441,79$ \\
\hline $\mathbf{1 9 5 3}$ & $4.488,45$ & $6.358,63$ & $2.943,37$ & $6.385,16$ \\
\hline $\mathbf{1 9 5 4}$ & $5.837,67$ & $12.196,30$ & 550,03 & $6.935,19$ \\
\hline $\mathbf{1 9 5 5}$ & $19.115,59$ & $31.311,89$ & $34.214,55$ & $41.149,74$ \\
\hline $\mathbf{1 9 5 6}$ & $67.078,28$ & $98.390,17$ & 136,40 & $41.286,14$ \\
\hline $\mathbf{1 9 5 7}$ & $44.684,50$ & $143.074,67$ & 291,18 & $41.577,32$ \\
\hline $\mathbf{1 9 5 8}$ & $105.779,45$ & $248.854,12$ & $14.925,37$ & 56.502 .69 \\
\hline $\mathbf{1 9 5 9}$ & $115.611,94$ & $364.466,06$ & $5.957,99$ & $62.460,68$ \\
\hline $\mathbf{1 9 6 0}$ & $30.167,52$ & $394.633,58$ & $4.504,03$ & $66.964,71$ \\
\hline $\mathbf{1 9 6 1}$ & $116.627,98$ & $511.261,56$ & $5.915,62$ & $72.880,33$ \\
\hline $\mathbf{1 9 6 2}$ & $319.600,30$ & $830.861,86$ & $106.515,32$ & $179.395,65$ \\
\hline $\mathbf{1 9 6 3}$ & $405.918,33$ & $1.236 .780,10$ & 108860,52 & $288.256,17$ \\
\hline $\mathbf{1 9 6 4}$ & $480.687,78$ & $1.717 .467,80$ & $188.613,19$ & $476.869,36$ \\
\hline $\mathbf{1 9 6 5}$ & $118.133,83$ & $1.835 .601,60$ & $61.685,19$ & $538.554,55$ \\
\hline $\mathbf{1 9 6 6}$ & $161.888,24$ & $1.997 .489,80$ & $74.717,96$ & $613.272,51$ \\
\hline $\mathbf{1 9 6 7}$ & $51.543,25$ & $2.049 .033,00$ & $31.869,55$ & $645.142,06$ \\
\hline $\mathbf{1 9 6 8}$ & $242.790,47$ & $2.291 .823,40$ & $55.460,37$ & $650.682,43$ \\
\hline $\mathbf{1 9 6 9}$ & $371.309,44$ & $\mathbf{2 . 6 6 3 . 1 3 2 , 8 0}$ & $103.452,84$ & $\mathbf{6 6 1 . 0 2 5 , 2 7}$ \\
\hline & & & & \\
\hline
\end{tabular}

Fuente: Archivo del Ayuntamiento de Benidorm, Licencias de Obras, 1951-1969. Elaboración propia. 
ción de hoteles de poca envergadura también es importante. Los promotores son agentes que tan sólo han solicitado una licencia de obras, aunque en algunos casos con estas obras han logrado capitalizarse y acometer actuaciones mayores. Las parcelas de dimensiones por encima de los $800 \mathrm{~m}^{2}$ se destinan a la construcción de los edificios residenciales y de apartamentos mayores, así como para algún hotel de mayor dimensión. Pero este tipo de construcciones no empiezan a ser frecuentes hasta el año 1957. También son utilizadas para la construcción de chalets en la zona exterior del planeamiento, ya que son necesarios, hasta el cambio de ley, un mínimo $10.000 \mathrm{~m}^{2}$ de solar para edificar con uso residencial.

A partir de 1960 el mayor número de solicitudes se centrará en los solares con más de $800 \mathrm{~m}^{2}$, con 370 expedientes. El número de solicitudes para las parcelas menores de $800^{2}$ será de 237. La construcción en solares pequeños se empieza a sustituir por promociones a cargo de promotores profesionales en solares de mayor capacidad. Sin embargo, aún es importante la construcción en solares de entre 101 y $200 \mathrm{~m}^{2}$, debido a que la construcción de edificios de pocas plantas y reducido número de viviendas se sigue produciendo en las cercanías del casco histórico. El mayor número de expedientes lo recogen las parcelas con

\section{Cuadro 5}

BENIDORM (1951-1969). NÚMERO DE EXPEDIENTES DE NUEVA PLANTA POR AÑO SEGÚN SUPERFICIE DEL SOLAR $\left(\mathrm{m}^{2}\right)$

\begin{tabular}{|l|c|c|c|c|c|c|c|}
\hline Año & $-\mathbf{1 0 0}$ & $\mathbf{1 0 1 - 2 0 0}$ & $\mathbf{2 0 1 - 4 0 0}$ & $\mathbf{4 0 1 - 8 0 0}$ & $\mathbf{8 0 1 - 1 . 2 0 0}$ & $\mathbf{1 . 2 0 1 - 2 . 0 0 0}$ & $\mathbf{+ 2 0 0 1}$ \\
\hline $\mathbf{1 9 5 1}$ & - & 3 & 2 & - & - & - & - \\
\hline $\mathbf{1 9 5 2}$ & 3 & 2 & 1 & 1 & - & - & - \\
\hline $\mathbf{1 9 5 3}$ & 1 & 1 & - & 3 & - & - & 1 \\
\hline $\mathbf{1 9 5 4}$ & 2 & 9 & 2 & 5 & 2 & - & - \\
\hline $\mathbf{1 9 5 5}$ & 4 & 10 & 3 & 2 & 7 & 3 & 1 \\
\hline $\mathbf{1 9 5 6}$ & 6 & 10 & 1 & 3 & 7 & 4 & 6 \\
\hline $\mathbf{1 9 5 7}$ & 3 & 10 & 3 & - & 15 & 5 & 2 \\
\hline $\mathbf{1 9 5 8}$ & 4 & 8 & 3 & 1 & 16 & 7 & 6 \\
\hline $\mathbf{1 9 5 9}$ & 5 & 6 & 2 & 1 & 6 & 6 & 11 \\
\hline $\mathbf{1 9 6 0}$ & - & 4 & 2 & 1 & 8 & 5 & 3 \\
\hline $\mathbf{1 9 6 1}$ & 7 & 10 & 3 & 3 & 9 & 6 & 14 \\
\hline $\mathbf{1 9 6 2}$ & 7 & 11 & - & 6 & 15 & 11 & 25 \\
\hline $\mathbf{1 9 6 3}$ & 19 & 30 & 10 & 12 & 16 & 8 & 23 \\
\hline $\mathbf{1 9 6 4}$ & 13 & 35 & 14 & 4 & 16 & 12 & 43 \\
\hline $\mathbf{1 9 6 5}$ & 6 & 9 & 3 & 2 & 2 & 4 & 11 \\
\hline $\mathbf{1 9 6 6}$ & 3 & 5 & 3 & 2 & 6 & 4 & 19 \\
\hline $\mathbf{1 9 6 7}$ & 1 & - & - & 2 & 4 & 5 & 9 \\
\hline $\mathbf{1 9 6 8}$ & - & 4 & 1 & 4 & 14 & 6 & 29 \\
\hline $\mathbf{1 9 6 9}$ & 2 & 4 & 3 & 2 & 13 & 7 & 39 \\
\hline Total & 86 & 171 & 56 & 54 & 156 & 93 & 242 \\
\hline
\end{tabular}

Fuente: Archivo del Ayuntamiento de Benidorm, Licencias de Obras, 1951-1969. Elaboración propia. 
más de $2.000 \mathrm{~m}^{2}$, en su mayoría para chalets en la zona periférica y sobre todo para promociones inmobiliarias mayores (en muchos casos se llegan a edificar más de 400 apartamentos).

Para el total del periodo (1951-1969) los solares con mayor número de solicitudes han sido los que miden más de $2001 \mathrm{~m}^{2}$. Los solares entre 101 y $200 \mathrm{~m}^{2}$ han sido los segundos más numerosos con 171 expedientes, mientras que los terceros en número han sido los solares entre 801 y $1.200 \mathrm{~m}^{2}$. La creciente importancia de los solares grandes nos demuestra la intervención de promotores externos con mucha capacidad de actuación, así como la creciente capitalización de algunos promotores locales.

b) Superficie construida ampliada

La superficie construida en el parque inmobiliario existente a través de los expedientes de adición (cuadro 6) ha experimentado cambios importantes en el periodo (1951-1969). El aumento hasta $100 \mathrm{~m}^{2}$ ha sido el más importante a lo largo de todo el periodo (95

Cuadro 6

BENIDORM (1951-1969). NÚMERO DE EXPEDIENTES DE ADICIÓN POR AÑO SEGÚN SUPERFICIE CONSTRUIDA AMPLIADA

\begin{tabular}{|l|c|c|c|c|c|c|c|}
\hline Año & $\mathbf{0 - 1 0 0}$ & $\mathbf{1 0 1 - 2 0 0}$ & $\mathbf{2 0 1 - 4 0 0}$ & $\mathbf{4 0 1 - 8 0 0}$ & $\mathbf{8 0 1 - 1 . 2 0 0}$ & $\mathbf{1 . 2 0 1 - 2 . 0 0 0}$ & $+\mathbf{2 . 0 0 1}$ \\
\hline $\mathbf{1 9 5 1}$ & 3 & - & - & - & - & - & - \\
\hline $\mathbf{1 9 5 2}$ & 3 & - & - & - & - & - & - \\
\hline $\mathbf{1 9 5 3}$ & - & - & - & - & - & - & - \\
\hline $\mathbf{1 9 5 4}$ & 2 & 3 & - & - & - & - & - \\
\hline $\mathbf{1 9 5 5}$ & 2 & 2 & 1 & 1 & - & - & - \\
\hline $\mathbf{1 9 5 6}$ & - & - & - & - & - & - & - \\
\hline $\mathbf{1 9 5 7}$ & 1 & - & - & - & - & - & - \\
\hline $\mathbf{1 9 5 8}$ & 1 & - & - & - & - & - & - \\
\hline $\mathbf{1 9 5 9}$ & - & - & - & - & - & - & - \\
\hline $\mathbf{1 9 6 0}$ & 9 & - & 1 & 1 & - & - & - \\
\hline $\mathbf{1 9 6 1}$ & 15 & 2 & 2 & 1 & - & - & - \\
\hline $\mathbf{1 9 6 2}$ & 16 & 4 & 2 & 2 & 1 & - & - \\
\hline $\mathbf{1 9 6 3}$ & 13 & 10 & 5 & 2 & - & 1 & 1 \\
\hline $\mathbf{1 9 6 4}$ & 13 & 10 & 3 & 2 & - & - & 1 \\
\hline $\mathbf{1 9 6 5}$ & 3 & 1 & 3 & 1 & 1 & 1 & - \\
\hline $\mathbf{1 9 6 6}$ & 5 & 2 & 2 & 3 & - & - & - \\
\hline $\mathbf{1 9 6 7}$ & 4 & 4 & - & - & - & - & 1 \\
\hline $\mathbf{1 9 6 8}$ & 3 & 1 & 1 & 3 & 2 & 2 & - \\
\hline $\mathbf{1 9 6 9}$ & 2 & 2 & 1 & 4 & 1 & 1 & 3 \\
\hline $\mathbf{T o t a l}$ & 95 & 41 & 21 & 20 & 5 & 5 & 6 \\
\hline
\end{tabular}

Fuente: Archivo del Ayuntamiento de Benidorm, Licencias de Obras, 1951-1969. Elaboración propia. 
solicitudes), con un número elevado de solicitudes en los años 1961 a 1964 (15, 16 y 13 expedientes respectivamente). En menos ocasiones se solicitan ampliaciones de entre 101 y $200 \mathrm{~m}^{2}$ (41 solicitudes), entre 201 y $400 \mathrm{~m}^{2}$ (21 solicitudes) y entre 401 y $800 \mathrm{~m}^{2}$ (20 solicitudes), mientras que raramente se solicitan proyectos de ampliación de entre 801 y $1.200 \mathrm{~m}^{2}$ (5 solicitudes), entre 1.201 y $2.000 \mathrm{~m}^{2}$ (5 solicitudes) y en más de $2.000 \mathrm{~m}^{2}$ (6 solicitudes). Estos últimos intervalos aparecen sobre todo al final del periodo (a partir de 1962) y crear anexos a los medios productivos turísticos ha sido su función principal. Hasta entonces las ampliaciones se realizan sobre todo a través del aumento de uno o como mucho dos plantas, lo que arroja totales de superficie construida muy bajos.

c) Densidad de construcción en los solares

Comparando la superficie del solar con la superficie construida podemos comprender la fuerte presión urbanística que empiezan a sufrir muchos solares en Benidorm (Cuadro 7). El mayor número de solicitudes se recoge en los solares entre 101 y $200 \mathrm{~m}^{2}$ y en los solares de más de $2.000 \mathrm{~m}^{2}$. Entre ambos existen grandes diferencias. Mientras que en los primeros la mayor parte de las solicitudes son para superficies construidas mayores de $100 \mathrm{~m}^{2} \mathrm{y}$ menores de $2.000 \mathrm{~m}^{2}$ (30 solicitudes de entre 101 y $500 \mathrm{~m}^{2}, 32$ entre 501 y $1.000 \mathrm{~m}^{2}$ y 21 entre 1.001 y $2.000 \mathrm{~m}^{2}$, en total 83 solicitudes), en el segundo caso (solares de más de $\left.2.000 \mathrm{~m}^{2}\right)$ son importantes las solicitudes con superficie construida entre 101 y $500 \mathrm{~m}^{2}$ (22 solicitudes) y las de más de $2.000 \mathrm{~m}^{2}$ (40 solicitudes). En el primer caso las superficies construidas son iguales o triplican la superficie de la parcela, mientras que en el segundo caso o es mucho menor o la supera en poco. Aún así, las parcelas de más de $2.000 \mathrm{~m}^{2}$ se utilizan para la construcción de grandes complejos residenciales y de apartamentos, pero en este periodo aún son poco numerosas. Son precisamente las parcelas más pequeñas las más cercanas al casco histórico, localizadas en el ensanche del casco, donde se construye con grandes densidades edificatorias. Las parcelas más alejadas del casco, habitualmente con funciones agrícolas, abandonan estas actividades progresivamente para especular. Son además las parcelas más grandes, que pueden ser adquiridas en ese periodo por bajos

Cuadro 7

BENIDORM (1951-1969). NÚMERO DE EXPEDIENTES DE NUEVA PLANTA SEGÚN SUPERFICIE DEL SOLAR Y SUPERFICIE CONSTRUIDA

\begin{tabular}{|l|c|c|c|c|c|c|}
\hline \multicolumn{7}{|c|}{ Superficie construida $\left(\mathbf{m}^{2}\right)$} \\
\hline Superficie solar $\left(\mathbf{m}^{2}\right)$ & $\mathbf{- 1 0 0}$ & $\mathbf{1 0 1 - 5 0 0}$ & $\mathbf{5 0 1 - 1 . 0 0 0}$ & $\mathbf{1 . 0 0 1 - 2 . 0 0 0}$ & $\mathbf{+ 2 . 0 0 1}$ & Total \\
\hline $\mathbf{0 - 1 0 0}$ & 13 & 31 & 1 & 0 & 0 & 44 \\
\hline $\mathbf{1 0 1 - 2 0 0}$ & 7 & 30 & 32 & 21 & 6 & 96 \\
\hline $\mathbf{2 0 1 - 4 0 0}$ & 1 & 8 & 6 & 13 & 3 & 31 \\
\hline $\mathbf{4 0 1 - 8 0 0}$ & 2 & 6 & 1 & 4 & 13 & 26 \\
\hline $\mathbf{8 0 0 - 1 . 2 0 0}$ & 3 & 37 & 5 & 6 & 6 & 57 \\
\hline $\mathbf{1 . 2 0 1 - 2 . 0 0 0}$ & - & 19 & 2 & 7 & 7 & 35 \\
\hline$+\mathbf{2 . 0 0 0}$ & 5 & 22 & 14 & 5 & 40 & 86 \\
\hline Total & 31 & 153 & 61 & 56 & 75 & 375 \\
\hline
\end{tabular}

Fuente: Archivo del Ayuntamiento de Benidorm, Licencias de Obras, 1951-1969. Elaboración propia. 
precios. La mayor parte de la construcción en estas parcelas corresponde a chalets y a casas de pocas plantas, de ahí la importancia de las solicitudes con superficies construidas entre 101 y $500 \mathrm{~m}^{2}$. En el resto de cruces entre superficie del solar y superficies construidas se dan situaciones tanto de uno como del otro caso.

\section{Conclusión}

El periodo de tiempo desde 1950 a 1970 es fundamental para entender la morfología urbana de Benidorm, ya que los cambios que tuvieron lugar han sido muy importantes en la consolidación de la ciudad actual. Estas transformaciones están relacionadas con los cambios propuestos en el ámbito político, así como en el cambio de modelo de ciudad, desde una ciudad jardín con vistas a un turismo residencial, a una ciudad destinada al turismo de masas. En este sentido, cabe destacar los siguientes puntos:

- Distinción de tres modelos en la solicitud de expedientes de obras: a) un modelo de construcción de ciudad cerrado (hasta 1956), con predominio de la reforma y ampliación de los hogares frente a la construcción de obra nueva; b) modelo de transición (hasta 1963) caracterizado por un incremento de la obra nueva destinada a la construcción de viviendas unifamiliares, se convierten así en el tipo de licencia predominante frente a la reforma o la ampliación; c) modelo actual (a partir de 1963) de construcción de ciudad, donde predomina la petición de obra nueva para la construcción de grandes conjuntos frente a la obra nueva de pequeñas dimensiones y la reforma o ampliación.

- Un cambio en el modelo de construcción de ciudad acorde con un cambio en la organización social y el sistema productivo. Podemos diferenciar tres momentos clave: a) una organización social basada en la estructura productiva agraria minifundista (hasta 1960), con carácter emigratorio, donde predomina la construcción por parte del futuro usuario de la vivienda, es decir, se trata de una construcción de ciudad familiar; b) el sector productivo principal empieza a estar representado por el turismo (hasta 1970), pero de carácter residencial, con pequeños movimientos inmigratorios, lo que da lugar a una promoción destinada principalmente a la construcción de chalets y viviendas unifamiliares secundarias, también se solicitan un número de licencias reducido destinadas a edificios residenciales para los inmigrantes laborales; c) una ciudad organizada social y espacialmente hacia un turismo de masas (a partir de 1970), con existencia de importantes flujos inmigratorios donde predomina la promoción inmobiliaria con vistas a la creación de mercancías, ya sean grandes conjuntos residenciales destinados a la inmigración de carácter laboral o grandes conjuntos turísticos (apartamentos, urbanizaciones, hoteles, etc.).

- La ciudad anterior a 1950, de tipo laxo y poca altura, tiene continuación en una ciudad abierta de tipo turístico-residencial. Ambas situaciones se ven progresivamente sustituidas por la creación de una ciudad densa y continua con un crecimiento principalmente en altura.

- El consumo de suelo se incrementa, debido a un aumento de la superficie de los solares utilizados para la construcción. Esto es debido principalmente a la importancia creciente de la gran promoción comercial frente a la pequeña de uso propio. Así mismo, debido a estos cambios en la promoción, la superficie construida no deja de crecer. Todo esto da lugar a un aumento general de la densidad constructiva en el municipio, explicada tanto por el aumento del valor del suelo como por la actuación de promotores foráneos profesionalizados cuyo objetivo principal es la búsqueda de beneficio. 


\section{Bibliografía}

ARRIOLA, Pedro $\mathrm{M}^{\mathrm{a}}$., (1991), La producción de una ciudad-máquina del capital: Vitoria-Gasteiz, Universidad del País Vasco, Bilbao.

AYUNTAMIENTO DE BENIDORM, (1955), Ordenanzas de construcción.

CAPEL, Horacio, (1975), Capitalismo y morfología urbana en España, Col. Realidad Geográfica, núm. 4, Los libros de la frontera, Barcelona, 142 pp.

CASTELLS, Manuel (1974), La cuestión urbana, Ed. Siglo XXI, Madrid.

COSTA, José (1997), «Interacción y conflicto entre estructura urbana y uso comercial. El caso de Benidorm», en Dinámica Litoral-Interior, Vol. I, Asociación de Geógrafos Españoles, Departamento de Xeografía da Universidade de Santiago de Compostela, pp. 313-321.

GAVIRIA, Mario, et al., (1977), Benidorm, ciudad nueva, Ed. Nacional, Madrid.

HARVEY, David (1977), Urbanismo y desigualdad social, Ed. Siglo XXI, Madrid.

INE, Censos de la población de España (1900, 1910, 1920, 1930, 1940, 1950, 1960, 1970, 1981, 1991, y 2001).

INE, Padrón municipal de habitantes de 1996.

MAZÓN, Tomás, (1994), La configuración del paisaje urbano en Alicante (1960-1990), Instituto de Cultura «Juan Gil-Albert», Diputación de Alicante, Alicante, 223 pp.

MAZÓN, Tomás (1997), Introducción a la planificación urbana, Ed. Aguaclara, Colección Amalgama, Alicante.

QUEREDA SALA, José, (1978), Comarca de la Marina, Alicante, Diputación provincial, Alicante.

ROSSELLÓ I VERGER, V. M., (1984), Cinquanta-cinc ciutats valencianes, Universidad de Valencia, Valencia.

SÁNCHEZ, J.E., (1981), La Geografía y el espacio social del poder, Col. Realidad Geográfica, num. 3, Los libros de la Frontera, Barcelona, 248 pp.

SÁNCHEZ, J.E., (1991), Espacio, economía y sociedad, Ed. Siglo XXI, Madrid.

SÁNCHEZ, J.E., (1992), Geografía Política, Ed. Síntesis, Madrid, 223 pp.

SORRIBES, Josep (1978), Crecimiento urbano y especulación en Valencia, Ed. Almudín, Valencia.

SORRIBES, Joseph (1985), Desarrollo capitalista y proceso de urbanización en el País Valenciano, Ed. Alfonso el Magnánimo, Valencia.

VERA REBOLLO, J.F. (1987), Turismo y urbanización en el litoral alicantino. Instituto de Cultura Juan Gil-Albert. Alicante, $431 \mathrm{pp}$.

VILAGRASA, Joan (1990), Creixement urbà i agents de la producció de l'espai: el cas de la ciutat de Lleida. 1940-1980, Institut Cartogràfic de Catalunya, Barcelona.

VILAGRASA, Joan (Ed.) (1997), Vivienda y promoción inmobiliaria en España, Universidad de Lleida, Colección espai/temps, Lleida. 
